\title{
Kommentare zu B. Röder und F. Rösler: Kompensatorische Plastizität bei blinden Menschen
}

Funktionelle kortikale Reorganisation versus Pseudoplastizität

\section{Thomas Elbert}

\section{Universität Konstanz}

Erfahrungen verändern unablässig und über die gesamte Lebensspanne hinweg die neuronalen Elemente. In der Folge unterliegen Hirnstruktur und -organisation einem permanenten Wandel. Mit Heraklit («bei einem Fluss ist es nicht möglich zweimal hinein $\mathrm{zu}$ steigen in denselben - auch nicht ein sterbliches Wesen zweimal zu berühren und zu fassen im gleichen Zustand ...») könnte man sagen, dass ein- und derselbe Reiz nicht ein zweites Mal in das gleiche Hirn Eingang finden kann. Erstaunlich ist dabei, dass vermehrter Gebrauch genauso wie ausbleibende Reizung eines Sinneskanals so starke Veränderungen im Gehirn bedingen können, dass diese auf der makroskopischen Ebene mit nicht-invasiven Methoden funktionell wie strukturell nachweisbar werden. Genauere Erkenntnisse, welchen Regeln diese makroskopische Neuroplastizität unterliegt, wie diese stimuliert oder unterbunden werden kann, welche Funktionsverbesserungen oder -beeinträchtigungen damit verknüpft sind, erweisen sich für die Neurorehabilitation als zunehmend bedeutsam, da darauf aufbauend neue therapeutische Bausteine, z. B. nach Schlaganfall entwickelt werden können und sich herkömmliche therapeutische Maßnahmen in ihrer Effizienz verbessern lassen (zusammenfassend z. B. Elbert et al., 2003, Elbert \& Heim, 2001).

Die Arbeiten von Röder und Rösler (2004) leisten anhand ihrer umfassenden und sorgfältigen Untersuchungen bei blinden Personen, also eines vom visuellen Eingang deprivierten Gehirns, einen ganz erhebli- chen Beitrag für unser Verständnis der Prozesse neuronaler Organisation und Reorganisation. Die besondere Bedeutsamkeit dieser Untersuchungen liegt einerseits in dramatischen Veränderungen von Funktionszuschreibungen zu Hirnregionen andererseits in der These, dass «geburtsblinde Menschen in auditiven Aufgaben ... bessere Leistungen als sehende Personen zeigen» (Röder \& Rösler, S. 255). Demnach kann selbst vergleichsweise elementare Reizverarbeitung auch außerhalb der klassischen zugeordneten sensorischen Repräsentationsareale durchgeführt werden und Rekrutierung solcher zusätzlicher Areale würde Funktionsverbesserung bedeuten.

In Ergänzung der Diskussion durch die Autoren selbst soll hier noch folgender Fragenkomplex beleuchtet werden:

1) Könnte es sich bei den berichteten Abweichungen in der funktionellen kortikalen Organisation von Blinden teilweise um eine Art «Pseudoplastizität» handeln, d.h. könnten manche Unterschiede sich einfach als Folge unterschiedlicher Aufmerksamkeit und unterschiedlicher Strategien in der Aufgabenbewältigung darstellen?

2) Sind die beobachteten Aktivierungen tatsächlich funktionell oder eher ein Epiphänomen, quasi wie der Lärm des laufenden Gehirnmotors?

3) Könnte eine minimale subkortikale Änderung in den Verbindungen zu den beobachteten makroskopischen Veränderungen im Kortex geführt haben? 
Ad 1) Eine wesentliche Frage, die sich generell bei Berichten über Variationen in funktionellen Hirnkarten stellt, ist diejenige, ob es sich um Neuroplastizität oder möglicherweise nur um eine «Pseudoplastizität» handeln könnte. Mit Neuroplastizität sind strukturelle Veränderungen in der Netzwerkarchitektur gemeint, d.h. morphologische Veränderungen, wie etwa Generation synaptischer Spines, veränderte Konnektivität und Verzweigung der Neurone oder Veränderung in Glia- und Neuronenzahl mit nachfolgender Angiogenese in den aktivierten Arealen. Solche Veränderungen sind nicht ohne weiteres, zumindest nicht instantan erzielbar und nicht ohne weiteres umkehrbar. Pseudo- plastizität hingegen bezeichnet die Schärfung oder Akzentuierung von Aktivierungsverteilungen durch kontextuelle Variablen oder Verschiebungen des Aufmerksamkeitsfokus. In diesem Fall bleibt das anatomische Substrat im Repräsentationsareal morphologisch unverändert. Es ändern sich nur modulierende Eingänge, «top-down» (Aufmerksamkeit, Motivation, Kontext) oder «bottom-up» (Aufgabenvariation). Nicht jede Veränderung in kortikalen Repräsentationsgebieten darf als Neuroplastizität interpretiert werden. So demonstrierten beispielsweise Braun et al. (2002), dass die homunkuläre Handrepräsentation im somatosensorischen Kortex allein durch Aufmerksamkeit in ihren Erscheinungsformen moduliert werden kann. Ein und derselbe Reiz, der über Finger und Hand hinweg strich führte zu einer Trennung der Fingerrepräsentationen, wenn die Reize nur an einem Finger, aber zu einer Fusion, wenn die Reize an der ganzen Hand aufgabenrelevant waren. Demnach modulierte der Kontext die homunkuläre Aktivierungsstruktur. Versuchspersonen, die zu einer solchen Modulation vergleichsweise gut in der Lage waren, erwiesen sich auch als vergleichsweise gut in den entsprechenden Aufgaben.

Wäre also denkbar, dass berichtete Unterschiede zwischen Blinden und sehenden Kontrollpersonen weniger Folge unterschiedlicher Hirnorganisation sondern aus unterschiedlichen Strategien in der Durchführung der Aufgaben etwa unterschiedlicher Aufmerksamkeit auf die Reize oder anderer Motivationslage resultieren könnten? Es erscheint schwierig einen solchen Einfluss durchweg auszuschließen.

Ad 2) Dennoch legen Röder und Rösler eine Reihe sehr starker Belege vor, dass die beobachteten Aktivierungen tatsächlich Verarbeitung außerhalb der klassischen Areale nahe legen. Wird etwa von blinden Personen verlangt, subtile Lautveränderungen $z u$ entdecken, so zeigt sich - im Gegensatz zu Sehenden - eine Aktivität auch in okzipitalen Regionen des Kortex, also Bereichen, die bei Sehenden ausschließlich visueller Verarbeitung vorbehalten bleiben. Ergänzend zu den Untersuchungen von Röder und Rösler erscheint mir aber das stärkste Argument in folgender Beobachtung zu liegen: Stört man die okzipitale Aktivität über magnetische Impulse, die mittels magnetischer Stimulation (TMS) spezifisch im Okzipitallappen verabreicht werden, so werden bei Blinden Leistungen beeinträchtigt, die bei Sehenden nur durch TMS-Impulse in anderen Regionen gestört werden können. Etwa verzerren okzipitale TMS-Impulse bei Blinden das Lesen der Blindenschrift oder führen zu Auslassungen von Buchstaben im Braille-Text (Cohen et al., 1997).

Einen interessanten experimentellen Ansatz haben Sur und Mitarbeiter (Sur et al., 1988; Sharma et al., 2000) im Verlauf des letzten Jahrzehnts perfektioniert. - Dieser erlaubt es, die sensorischen Nervenbahnen, die in die verschiedenen Kortexareale verlaufen, bei jungen Frettchen in andere Areale quasi «umzubahnen». Genauer gesagt, werden die neuronalen Bahnen aus der Retina, die normalerweise über den visuellen Thalamus zum primären visuellen Kortex (V1) verlaufen, umgeleitet, sodass sie nun in den auditorischen Thalamus münden, der seinerseits den primären auditorischen Kortex (A1) innerviert. Der Thalamus bleibt dabei von seinen normalen auditorischen Eingängen depriviert. Damit kann Organisations- und Reaktionsweise kortikalen Gewebes, also die Selbstorganisation des Kortex in Abhängigkeit der korrelierten Feuerungsmuster aus den Eingängen studiert werden. Interessanterweise prägt sich bei diesen Tieren in A1 eine für den visuellen Kortex typische Organisation aus, etwa findet sich die charakteristische «Pinwheel»-Struktur, nach der verschiedene Orientierungen eines visuellen Reizes in Form eines Windrades auf der Kortexoberfläche repräsentiert sind, nun im akustischen Kortex wieder. Von Melchner et al. (2000) belegten, dass die Versuchstiere auf visuelle Reize adäquate Verhaltensweisen zeigen, d. h., dass sie mit ihrem auditorischen Kortex tatsächlich «sehen» können. Die sensorischen Eigenschaften, die mit einer bestimmten Modalität verknüpft sind, werden also offensichtlich dadurch bestimmt, wie zeitsynchrone Eingänge die funktionelle Organisation des Kortex ausprägen.

Ad 3): Für intermodale Plastizität berichten Röder und Rösler bei. blinden Personen Funktionsverschiebungen, die sich über ganze kortikale Lappen hinweg also über mehrere $\mathrm{cm}$ hinweg bewegen können. Elbert et al. (1994) beobachteten erstmals beim Menschen eine ausgedehnte Reorganisation der somatosensorischen Repräsentationsareale nach Amputation des Armes, die gleichfalls Verschiebungen im $\mathrm{cm}$-Bereich beinhalteten: Neurone der «deafferenzierten» Areale von Hand und Arm reagieren auf Eingänge in benachbarte Repräsentationsareale also nach Stimulation von Schulter und Gesicht. Ähnliche großräumige Verschiebungen waren zuvor von Pons et al. (1991) bei Affen beobachtet worden. Intra- wie intermodale Ver- 
schiebungen können demnach mehrere Zentimeter betragen. Damit können Erklärungen, die eine Sprossung des Dendritenbaumes oder der Axonendigungen als einzigen Ursprung von Reorganisation vermuteten, nicht ausreichen, vielmehr ist eine Veränderungen in weit verzweigten neuronaler Verbindungen, d. h. eine makroskopische kortikale Reorganisation erforderlich, um dieses Phänomen zu erklären. Alternativ könnte man annehmen, dass eine mikroskopische Umorganisation auf subkortikaler, etwa thalamischer Ebene sich in den nachgeschalteten kortikalen Arealen makroskopisch auswirken könnte. Systematische Untersuchungen auf den verschiedenen Ebenen konnten dies als einzige Erklärung jedoch ausschließen. So demonstrierten Ergenzinger et al. (1998), dass sich die thalamische Organisation deutlich verändert, wenn die efferenten Fasern vom Kortex zum Thalamus ausgeschaltet werden. Demnach wird die Reorganisation auf früheren Schaltstationen entlang der sensorischen Bahnen über «Top-down»-Prozesse entscheidend beeinflusst. Die funktionelle Organisation einer Ebene wird im Wechselspiel mit den vor- und nachgeschalteten Repräsentationen realisiert. Dabei dominiert kortikale Organisation wohl aus zwei Gründen: Sie ist die am Umfassendsten strukturierte und erlaubt aufgrund der im Kortex ausgeprägten lateralen Inhibition die größte Schärfe, d. h. die feinste Auflösung rezeptiver Felder. Zehnmal mehr Fasern verlaufen vom Kortex zum Thalamus, als umgekehrt vom Thalamus zum Kortex projizieren. Dadurch kann die Feinstruktur der kortikalen Organisation auch die funktionelle Organisation auf thalamischer Ebene mitbestimmen, d.h. letztlich bestimmt die kortikale funktionelle Organisation, wie Information bereits auf der ihr vorgeschalteten Ebene aufgearbeitet und strukturiert wird.

Wenn wir festhalten, dass der auditorische Kortex visuelle Information verarbeiten kann und umgekehrt, bei Blinden akustische Information im visuellen Kortex mit verarbeitet wird, wenn darüber hinaus der Informationseingang schon durch top-down Filter in den Kortex wesentlich mit bestimmt wird, dann stellt sich die Frage, warum dieses offensichtlich enorme Potenzial an Neuroplastizität nicht besser in der Neurorehabilitation genutzt werden kann. Dagegen lässt sich natürlich zuerst anführen, dass Funktionserholung in den meisten Fällen in einem ganz erstaunlichen Ausmaß erfolgt. Man stelle sich dazu eine beliebige vom Menschen gemachte Informationen verarbeitende Maschine vor, bei der eine lokale Zerstörung erfolgt ist. Dies führt in der Regel unwiederbringlich zum kompletten Funktionsausfall.

Ein weiterer wichtiger Aspekt wurde aus den Arbeiten von Rösler und Röder deutlich, Neuroplastizität und die Fähigkeit zu kortikaler Reorganisation hängt von den Phasen menschlicher Entwicklung ab und ist im sich entwickelnden Gehirn (Geburtsblinde) deut- lich höher als im Erwachsenenalter. Unbekannt ist gegenwärtig noch, in welchem Altersabschnitt die sensiblen Phasen kortikaler Reorganisation liegen. Die einstmals dominierende Sichtweise, dass pränatale und frühe postnatale Entwicklung ausschließlich durch eine starre Kaskade an genetischen Programmen bestimmt werden, wird heute nicht mehr vertreten. Erfahrung genauso wie Verletzung haben dramatische Auswirkungen, die im sich entwickelnden $\mathrm{Ge}$ hirn bis hin $\mathrm{zu}$ veränderten neuroanatomischen Bahnen führen können (zusammenfassend Payne \& Lomber, 2001). Obwohl kognitive Entwicklung gut studiert ist, wissen wir bisher so gut wie nichts über die Wirkung von Erfahrungen und Läsionen auf die kognitive und perzeptive Leistungsfähigkeit. Röder \& Röslers Pionierarbeiten zeichnen sich schließlich auch dadurch aus, dass sie diese Leistungsfähigkeit in Abhängigkeit des Zeitpunkts der Erfahrungsänderung (Geburt, spät erblindet, keine Läsion) quantifizierten. Eine genaueres Verständnis wäre wünschenswert, auch um sich damit vielleicht einen Jungbrunnen fürs Alter zu erschließen. Denn letztlich bedeutet Altern ohne wesentliche Funktionsbeeinträchtigung die Ausbeutung der Neuroplastizität.

\section{Literatur}

Braun, C., Haug, M., Wieck, K., Birbaumer, N., Elbert, T. \& Roberts, L.E. (2002). Functional organization of primary cortex depends on the focus of attention. Neuroimage, 17, 1451-1458.

Cohen, L. G., Celnik, P., Pascual-Leone, A., Corwell, B., Falz, L., Dambrosia, J., Honda, M:, Sadato, N., Gerloft, C., Catala, M.D. \& Hallett, M. (1997). Functional relevance of cross-modal plasticity in blind humans. $\mathrm{Na}$ ture, 389, 180-183.

Elbert, T., Rockstroh, B., Bulach, D. \& Meinzer, M. (2003). Die Fortentwicklung der Neurorehabilitation auf verhaltensneurowissenschaftlicher Grundlage: Beispiel Cl-Therapie. Nervenarzt, 74, 334-342.

Elbert, T. \& Heim, S. (2001). Cortical reorganization, a light and a dark side. Nature, 411, 139.

Elbert, T., Flor, H., Birbaumer, N., Knecht, S., Hampson, S. \& Taub, E. (1994). Extensive reorganization of the somatosensory cortex in adult humans after nervous system injury. Neuroreport, 5, 2593-2597.

Ergenzinger, E.R., Glasier, M.M., Hahm, J.O. \& Pons, T.P. (1998). Cortically induced thalamic plasticity in the primate somatosensory system. Nature $\mathrm{Neu}$ roscience, 1, 226-229.

Melchner, L, Pallas, S.L. \& Sur, M. (2000). Visual behavior mediated by retinal projections directed to the auditory pathway. Nature, 404, 871-876.

Payne, B.R. \& Lomber, S. G. (2001). Reconstructing 
functional systems after lesions of cerebral cortex. $\mathrm{Na}$ ture Review Neuroscience, 2, 911-919.

Pons, T.P., Garraghty, P.E., Ommaya, A. K., Kaas, J. H., Taub, E. \& Mishkin, M. (1991). Massive cortical reorganization after sensory deafferentation in adult macaques. Science, 252, 1857-1860.

Röder, B. \& Rösler, F. (2004). Kompensatorische Plastizität bei blinden Menschen: Was Blinde über die Adaptivität des Gehirns verraten. Zeitschrift für Neuropsychologie, in dieser Ausgabe.

Sharma, J., Angelucci, A. \& Sur, M. (2000). Induction of visual orientation modules in auditory cortex. Nature, 404, 841-847.

Sur, M., Garraghty, P.E. \& Roe, A. W. (1988). Experimen- tally induced visual projections into auditory thatamus and cortex. Science, 242, 1437-1441.

Prof. Dr. Thomas Elbert

Universität Konstanz

FB Psychologie

Universitätsstr. 10

D-78457 Konstanz

Tel. +4931883363

Fax +49 31882891

E-mail thomas.elbert@uni-konstanz.de 